\title{
Non-genetic factors associated with the risk of Parkinson's disease in Iranian patients
}

\author{
Narges Hosseini Tabatabaei, MDa,b \\ Babak Babakhani, MDa \\ Azadeh Hosseini Tabatabaei, PharmDc \\ Zahra Vahabi, MD ${ }^{b}$ \\ Akbar Soltanzadeh, MD
}

\begin{abstract}
a Brain and Spinal Cord Injury Research Center, Neuroscience Institute, Tehran University of Medical Sciences, Tehran, Iran

b Department of Neurology, Shariati Hospital, Tehran University of Medical Sciences, Tehran, Iran

c Department of Surgery, University of British

Columbia, Vancouver, Canada
\end{abstract}

Correspondence to: Akbar Soltanzadeh

E-mail: aksoltan@yahoo.com

\section{Summary}

The aim of this study was to investigate some of nongenetic factors associated with Parkinson's disease (PD) in a sample of Iranian patients.

This case-control study included 75 patients with idiopathic PD and 75 control patients.

The patients in the control group were found to have drunk more glasses of tea per day than the case group before the onset of their problem $(p=0.019)$. Every extra glass of tea per day decreased the risk of $P D$ by 0.8 times (OR=0.8, 95\% $\mathrm{Cl}=0.73-0.97, \mathrm{p}=0.02$ ). Each cup of coffee per week decreased the chance of developing PD by 0.5 times $(\mathrm{OR}=0.5,95 \% \mathrm{Cl}=0.28-0.9$, $\mathrm{p}=0.021$ ). A previous history of evening work increased PD risk by 4.6 times (OR=4.6, $95 \% \mathrm{Cl}=1.29$ 16.86, $p=0.019$ ) while major stressful events increased it by 13.5 times $(O R=13.5,95 \% \mathrm{Cl}=4.7-38.1$, $\mathrm{p}=0.0001$ ).

In conclusion, coffee and tea consumption may exert a protective effect against $\mathrm{PD}$, while evening work and stress may be risk factors for the development of the disease.

KEY WORDS: case-control study, Parkinson's disease, protective factors, risk factors

\section{Introduction}

Parkinson's disease (PD) is a neurodegenerative disease with a prevalence of 360 per 100,000 and an incidence of 18 per 100,000 people (Jancovic and
Shannon, 2008). There is evidence that several environmental and genetic factors can, together, contribute to this disease.

One study evaluating the association between adiposity in middle age and the risk of PD (Abbott et al., 2002) showed that increased triceps skin fold thickness in midlife could increase the risk of PD, as might an increased body mass index (BMI) (Hu et al., 2006). Instead, an assessment of two large cohort studies failed to find an association between history of hypertension, diabetes and hypercholesterolemia and PD (Simon et al., 2007). One study showed reduced vascular disease risk factors in patients with PD compared with a control group (Scigliano et al., 2006), while the results of studies conducted by Semchuk and colleagues (Semchuck et al., 1992; Semchuck and Love, 1995) demonstrate that contact with pesticides can increase the risk of PD. Kamel et al. (2007) also showed that exposure to some pesticides could increase PD risk. An epidemiological study found that female patients with PD showed a higher prevalence of ovariectomy ( $14.1 \%$ of cases vs $8.5 \%$ of controls) and an earlier age at menopause $(\mathrm{OR}=2.18 ; 95 \%$ $\mathrm{Cl}=0.88-5.39$ ) (Benedetti et al., 2001). Furthermore, ovariectomy before menopause has been suggested to increase the risk of PD (Rocca et al., 2008). Studies investigating the effect of non-steroidal anti-inflammatory drugs (NSAIDs) on the incidence of PD have given different results, with some showing no obvious association (Samii et al., 2009; Hancock et al., 2007) and others showing a protective effect of aspirin (ASA) and other NSAIDs on PD (Wahner et al., 2007; Chen et al., 2003). Recent investigations suggest that caffeine and tea consumers have a lower risk of PD (Hernán et al., 2002; Ragonese et al., 2003). A recent study conducted on consumers of Asian coffee and caffeine-containing drinks revealed a protective effect of these beverages (Tanaka et al., 2011). A quantitative review seemed to show that tea consumption can protect against PD and that this effect is more obvious in the Chinese population (Barranco Quintana et al., 2009). Patients with PD have also been found to smoke fewer cigarettes than their healthy siblings (Scott et al., 2005). More pack years of cigarette smoking and older age at cigarette withdrawal seem to decrease the risk of PD (Thacker et al., 2007). Finally, patients with PD are more likely to have a history of trauma, possibly because of the associated progressive motor problems (Rugbjerg et al., 2008).

One study has suggested an association between long sleep duration and PD. It was suggested that habitual longer sleep duration could be an earlier 
marker of PD and that working on night shifts has a protective effect against PD (Chen et al., 2006).

The aim of this study was to assess the non-genetic factors associated with the development of PD in a group of patients drawn from the Iranian population.

\section{Materials and methods}

\section{Study subjects}

This case-control study included 150 individuals who were admitted to the Shariati Hospital in Tehran, Iran, between May 2007 and May 2008. The population comprised two groups: cases experiencing idiopathic PD $(n=75)$ and controls $(n=75)$ (Table I). The following criteria were used to diagnose idiopathic PD and thus to assign affected individuals to the case group: the presence of at least two signs among the following: rigidity, bradykinesia, postural instability and tremor; or the presence of only one of these signs together with improvement of other signs with use of antiparkinson medications. Patients were excluded from the study if they presented symmetric signs at the onset of the disease, dementia within the first year of onset of the parkinsonism, overt dementia, or a Mini-Mental State score of less than 24. Other exclusion criteria were: bulbar signs and symptoms, early gait disorder, falling within the first year of onset of the disease, wheelchair dependence within five years, early autonomic failure, sleep apnea, gasping respiration, apraxia, alien limb, cortical sensory loss, abnormal metabolic tests (including blood urea nitrogen, creatinine, calcium, magnesium, aspartate aminotransferase, alanine aminotransferase, alkaline phosphatase), abnormal Wilson's disease test results in patients younger than 50 years, a history of antipsychotic drug use, abnormal neuroimaging studies.

The control subjects, who were recruited from departments other than the neurology department, had no history of central nervous system disease, no history of diseases affecting fertility, and no probable consequences of preexisting diseases that could affect PD. Table II lists the reasons for the hospital admission of all the members of the control group. This study was approved by the ethics committees of Tehran University of Medical Sciences and the Shariati Hospital. All the participants were informed about the purpose of the study and assured that their identity would remain confidential.

\section{Data collection}

The participants were interviewed directly and questionnaires were filled in using the information collected. In both the controls and the cases, the information collected referred to the same temporal period, i.e. before the onset of the symptoms that resulted in hospitalization and before the onset of $\mathrm{PD}$, respectively. The participants were asked to answer questions regarding their demographics (age, sex, BMI), dietary habits (i.e. consumption of tea or coffee), smoking habits, and lifestyle, including nocturnal sleep duration. Information was also collected on evening work (i.e. whether or not they had worked evenings for at least one year prior to the onset of the PD or of the problem leading to their hospitalization), occupational toxin exposure, medication use, previous head traumas (i.e. head traumas causing concussion), and experience of major stressful events, i.e. serious enough to trigger the disease (for example, the death of a family member, job loss, divorce, accidents involving children, and number of pregnancies for female participants).

\section{Statistical analyses}

In order to calculate the minimum sample size, exposure to agricultural toxins was considered as a risk factor. Considering an alpha error equal to 0.05 , a beta error equal to 0.2 (power of $80 \%$ ), and assuming there to be exposure to agricultural toxins in $15 \%$ of the control group and estimating an OR equal to 3 , the minimum sample size required for each group was calculated as 75 subjects. The calculations were performed using Power and Sample Size Calculations, Version 2.1.30 software (Biostat, Engelwood, New Jersey).

Next, the members of the case and control groups were matched one-to-one according to sex and age ( \pm 2 years). PD patients were classified according to the modified Hoehn and Yahr scale (Jancovic and Shannon, 2008). After checking the normality of the data, quantitative and qualitative variables were compared and analyzed using Student's t-test and the chi-

Table I - Characteristics of Parkinson's disease cases and control subjects

\begin{tabular}{lll}
\hline Variables & \multicolumn{2}{c}{$n(\%)$} \\
& Cases $(n=75)$ & Controls $(n=75)$ \\
\hline Sex & & \\
$\quad$ male & $51(68 \%)$ & $51(68 \%)$ \\
$\quad$ female & $24(32 \%)$ & $24(32 \%)$ \\
Age in years & $67.5 \pm 2.64$ & $68.6 \pm 1.13$ \\
(mean \pm SD) & & \\
\hline
\end{tabular}

SD:standard deviation

Table II - Reasons for hospitalization of the controls $(n=75)$.

\begin{tabular}{ll}
\hline Reasons for hospitalization & $\mathrm{n}(\%)$ \\
\hline Neck of femur fracture & $15(20)$ \\
Colles' fracture & $13(17.33)$ \\
Knee joint arthroplasty & $24(32)$ \\
Appendicitis & $7(9.33)$ \\
Pancreatitis & $2(2.66)$ \\
Mesenteric ischemia & $1(1.33)$ \\
Acute cholecystitis & $4(5.33)$ \\
Acute diverticulitis & $8(10.66)$ \\
Bowel perforation & $1(1.33)$ \\
\hline
\end{tabular}


square test respectively. Statistical analyses were performed using SPSS software, version 17 (SPSS Inc., Chicago, Illnois, USA). Multivariate logistic regression analysis was used to analyze the data using a $p$-value threshold of $<0.2$

\section{Results}

Applying the various inclusion and exclusion criteria, this study was initially launched with 79 cases and 79 controls. However, one of the participants in the case group was at stage 4 of PD and due to physical limitations was not able to attend all the interviews for data collection; this participant was therefore excluded. In addition, three controls unwilling to continue collaborating because of their post-operative conditions were also excluded. The individuals matched with these subjects in the control and case groups were therefore excluded as well. As a result, 75 cases and 75 controls were enrolled in this study; each group contained $51(68 \%)$ males and $24(32 \%)$ females (Table I). As shown in Table III, the case group patients were classified according to the modified Hoehn and Yahr staging scale. Evaluating exposure to agricultural toxins, we found that $26(34.66 \%)$ individuals with PD and 17 $(22.66 \%)$ controls had a history of toxin exposure, however the Pearson chi-square test did not show this difference to be significant ( $p=0.104$, Table IV). As shown in table $\mathrm{V}$ (over), the univariate logistic regression analysis indicated that previous toxin exposure increased the chance of developing PD by 1.8 times $(\mathrm{OR}=1.8,95 \% \mathrm{Cl}=0.88-3.72, \mathrm{p}=0.106)$, but the multivariate logistic regression analysis showed that the effect of this factor in the presence of other factors was negligible.

The data showed that $14(18.70 \%)$ individuals from the case group and 26 (34.70\%) controls had used ASA before the onset of the disease, and the chi-square test showed this difference to be significant $(p=0.027)$ (Table IV). However, the univariate and multivariate logistic regression analyses suggested that the effect of this factor was negligible (Table V, over). On assessing NSAID consumption before the onset of the disease, it emerged that seven cases $(9.30 \%)$ and 14 controls $(18.70 \%)$ had used NSAIDs more than once a week for a period lasting at least one year. However, the difference between these values was found to be insignificant $(p=0.1)$ (Table IV). The univariate and multivariate studies showed the effect of NSAIDs on the onset or progression of PD to be negligible (Table V).

Two cases $(2.7 \%)$ and 15 controls $(20 \%)$ had a histo-

Table III - Classification of the cases according to the modified Hoehn and Yahr scale

\begin{tabular}{lll}
\hline Stage & $n$ & $\begin{array}{l}\text { Time from onset of PD, } \\
\text { in years (mean } \pm \text { SD) }\end{array}$ \\
\hline 0 & 0 & 0 \\
1 & 15 & $1.2 \pm 0.50$ \\
1.5 & 5 & $2.7 \pm 1.11$ \\
2 & 22 & $3.1 \pm 1.73$ \\
2.5 & 16 & $3.6 \pm 2.16$ \\
3 & 16 & $5.2 \pm 3.24$ \\
4 & 1 & 8 \\
\hline
\end{tabular}

Abbreviations: $\mathrm{n}=$ number of cases; $\mathrm{SD}=$ standard deviation

Table IV - Results of univariate analysis (chi-square and Student's t-test) of factors associated with PD

\begin{tabular}{llll}
\hline Variables & Cases & Controls & p-value \\
\hline BMI $\left(\mathrm{kg} / \mathrm{m}^{2}\right)$, mean \pm SD & $20.65 \pm 3.26$ & $21.96 \pm 2.51$ & 0.008 \\
Night sleep duration (hours), mean \pm SD & $7.57 \pm 1.39$ & $6.96 \pm 1.50$ & 0.011 \\
Tea consumption (glasses drunk per day), mean \pm SD & $3.16 \pm 3.26$ & $4.38 \pm 3.02$ & 0.019 \\
Coffee drinkers, $\mathrm{n}(\%)$ & $2(2.70)$ & $15(20)$ & 0.001 \\
Patients with toxin exposure, $\mathrm{n}(\%)$ & $26(34.66)$ & $17(22.66)$ & 0.104 \\
ASA users, $\mathrm{n}(\%)$ & $14(18.70)$ & $26(34.70)$ & 0.027 \\
NSAID users, $\mathrm{n}(\%)$ & $7(9.30)$ & $14(18.70)$ & 0.10 \\
Term pregnancies, mean number & 5.5 & 4.8 & 0.041 \\
Cigarette smokers, $\mathrm{n}(\%)$ & $11(14.70)$ & $12(16.00)$ & 0.82 \\
Patients with a history of evening work, $\mathrm{n}(\%)$ & $13(17.80)$ & $6(8.00)$ & 0.075 \\
Patients with stress, $\mathrm{n}(\%)$ & $39(53.40)$ & $8(10.70)$ & 0.0001 \\
Patients with stress due to a family death, $\mathrm{n}(\%)$ & $16(21.30)$ & $9(12.00)$ & 0.125 \\
Patients with previous head trauma, $\mathrm{n}(\%)$ & $14(18.70)$ & $16(21.30)$ & 0.683 \\
Patients with previous head trauma with unconsciousness, $\mathrm{n}(\%)$ & $7(9.3)$ & $3(4.00)$ & 0.190 \\
Patients with LDL levels $>160, \mathrm{n}(\%)$ & $12(16.70)$ & $10(13.30)$ & 0.59 \\
Patients with hypertriglyceridemia, $\mathrm{n}(\%)$ & $12(16.70)$ & $8(10.70)$ & 0.289 \\
Patients with hypertension, $\mathrm{n}(\%)$ & $24(32.00)$ & $22(29.30)$ & 0.723 \\
\hline
\end{tabular}

Abbreviations: $\mathrm{BMI}=$ body mass index, $\mathrm{SD}=$ standard deviation; $\mathrm{ASA}=$ aspirin; $\mathrm{NSAID=non-steroidal} \mathrm{anti-inflammatory} \mathrm{drug;}$ $\mathrm{LDL}=$ low-density lipoprotein; $\mathrm{n}=$ number 
ry of coffee consumption (more than one cup per week before the onset of the disease) and the difference in this variable between the two groups was statistically significant $(p=0.001)$ (Table IV). The multivariate analysis showed that each cup of coffee per week decreased the chance of developing PD by 0.5 times, thus revealing its protective effect $(\mathrm{OR}=0.51,95 \%$ $\mathrm{Cl}=0.28-0.90, \mathrm{p}=0.021$, Table VI).

Daily tea consumption before the onset of the disease was found to average 3.16 glasses in the cases and 4.38 glasses in the controls and the independent t-test analysis showed this difference between the groups to be significant $(p=0.019$, Table IV). On multivariate analysis it emerged that each glass of tea decreased the chance of developing PD by 0.8 times, showing that tea consumption is a protective factor against $P D$ (OR=0.84, 95\% $\mathrm{Cl}=0.739-0.975, \mathrm{p}=0.02$ ) (Table VI).

The data showed an average nocturnal sleep duration of 7.57 hours in the cases and 6.96 hours in the controls and the independent t-test analysis showed a marked difference between the case and control groups in this variable $(p=0.01$, Table IV). The multivariate analysis seemed to show that every extra hour of sleep compared to mean sleep duration increases the chance of PD by 1.38 times $(\mathrm{OR}=1.38,95 \% \mathrm{Cl}=1.009$ 1.910, $p=0.044)$ (Table VI).

Thirteen (17.8\%) cases and six $(8.00 \%)$ controls had a history of evening work ( $p=0.75$, Table IV). Our data revealed that a history of evening work before the onset of PD increased the risk of PD by 4.6 times (OR=4.6, 95\%Cl=1.29-16.86, $\mathrm{p}=0.019)$ (Table VI).
The cases had an average BMI of $20.65 \mathrm{~kg} / \mathrm{m}^{2}$, versus $21.94 \mathrm{~kg} / \mathrm{m}^{2}$ in the controls (Table IV). The independent t-test showed a significant difference between these values $(p=0.008)$.

The collected data showed that the women with PD had more term pregnancies than those in the control group (average of 5.5 versus 4.8 ) and this difference was statistically significant $(p=0.041)$ (Table IV).

Thirty-nine of the cases (53.4\%) and eight of the controls $(10.70 \%)$ had experienced major stressful events that, as described by the patients, could have been severe enough to trigger the disease. The ten most common stressful events were considered as variables. These were: death of a spouse, divorce or marital separation, imprisonment, death of a close family member, personal injury or illness, dismissal from work, marital reconciliation, retirement, and changes in health condition of family members (Holmes and Rahe, 1967). The Pearson chi-square test suggested a significant difference between the above values $(p=0.0001)$ (Table IV). The multivariate logistic regression showed that the probability of PD was increased by 13.5 times in individuals with stress exposure than in people not exposed to stress $(O R=13.51$, $95 \% \mathrm{Cl}=4.79-38.15, \mathrm{p}=0.0001$ ) (Table $\mathrm{VI}$ ). The data showed that $16(21.3 \%)$ patients in the case group and nine $(12 \%)$ controls had experienced the death of a family member. However, this difference was not statistically significant $(p=0.125)$ (Table IV). The results after multivariate analysis suggested that the role of this kind of stress in causing PD was negligible.

Table V - Results of univariate logistic regression analysis of factors associated with PD.

\begin{tabular}{lllll}
\hline Variables & p-value & Odds Ratio & \multicolumn{2}{c}{$95 \% \mathrm{Cl}$ for Odds Ratio } \\
& & & Upper & 3.72 \\
\hline Toxin exposure & 0.106 & 1.8 & 0.88 & 2.55 \\
ASA use & 0.92 & 0.95 & 0.35 & 4.08 \\
NSAID use & 0.74 & 1.22 & 0.36 & 0.96 \\
Tea consumption & 0.01 & 0.82 & 0.71 & 2.00 \\
Sleep hours & 0.02 & 1.45 & 1.05 & 35.22 \\
Major stress & 0.0001 & 11.79 & 3.94 & 8.26 \\
Stress due to a family death & 0.25 & 2.16 & 0.57 & 21.79 \\
History of evening work & 0.4 & 5.56 & 1.42 & 0.93 \\
Coffee consumption & 0.02 & 0.53 & 0.30 & 5.16 \\
Cigarette smoking & 0.19 & 1.02 & 0.71 &
\end{tabular}

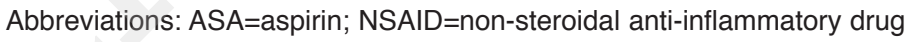

Table VI - Results of multivariate logistic regression analysis of factors associated with PD.

\begin{tabular}{lllll}
\hline Variables & Odds Ratio & \multicolumn{2}{l}{$95 \% \mathrm{Cl}$ for Odds Ratio } & U-value \\
& & Lower & 0.975 & 0.02 \\
\hline Tea consumption & 0.849 & 0.739 & 1.910 & 0.044 \\
Sleep hours & 1.38 & 1.009 & 38.15 & 0.0001 \\
Stress & 13.51 & 4.79 & 16.86 & 0.019 \\
Evening work & 4.67 & 1.29 & 0.904 & 0.021 \\
Coffee consumption & 0.51 & 0.28 & & \\
\hline
\end{tabular}


Comparison of smoking habits before onset of the disease showed that 11 cases (14.70\%) versus 12 controls $(16 \%)$ had smoked more than one-pack year of cigarettes. This difference was not significant $(p=0.82)$ (Table IV).

A history of head trauma was found in $14(18.7 \%)$ cases and in $16(21.3 \%)$ controls. However, the difference between these values was not significant on analysis with the Pearson chi-square test $(p=0.19)$.

Finally, our data showed that 24 cases (32\%) and 22 controls $(29.3 \%)$ had hypertension, and the difference between these two values was not significant $(p=0.72)$. Analysis of the results of the blood studies also failed to reveal significant differences: 12 cases (16\%) and 8 controls $(10.70 \%)$ had triglyceride levels $>200$ $(p=0.28)$, while 12 cases $(16.70 \%)$ and 10 controls (13.30\%) had LDL levels >160 ( $p=0.59)$ (Table IV).

\section{Discussion}

Parkinson's disease, which is often incapacitating, is the second most common neurodegenerative disorder after Alzheimer's disease (Wirdefeldt et al., 2011), with a growing prevalence accompanying the global aging of the population. The etiology of PD is complex, involving both genetic and environmental factors. In this study, we evaluated the role of some environmental factors in the initiation or progression of PD.

The results of this study show that a history of stress has a noticeable role in the initiation of PD. Although the stress deriving from the loss of a family member was not found to have a significant effect on the onset of the disease, other major stressful events, such as bankruptcy, job loss, divorce, and accidents involving children, were found to play a very important role.

It is hypothesized, partly on the strength of the lower incidence and prevalence of the disease in women compared to men, that estrogen has a protective role in PD, possibly linked to antioxidant and neuroprotective effects of estrogen on dopaminergic neurons (Shulman, 2002). Previous studies have demonstrated that an early reduction in endogenous estrogen (e.g. caused by ovariectomy in younger women) results in an increased risk of PD (Benedetti et al., 2001; Rocca et al., 2008). The women with PD in our sample had significantly more term pregnancies than the controls and pregnancy is, of course, a period of estrogen deprivation.

The effect of NSAIDs on PD is quite controversial. A number of previous studies have reported that regular use of non-ASA NSAIDs decreases the risk of PD. However no association was reported for ASA and acetaminophen (Chen et al., 2003; Wahner et al., 2007). Meanwhile one family-based case-control study (Hancock et al., 2007) and two populationbased case-control studies (Bower et al., 2006; Ton et al., 2006) reported no association between ASA or NSAIDs and PD. The data collected in our sample showed that the PD patients had used less ASA than the control group before the onset of their problem, a result that is in line with the findings of others (Wahner et al., 2007). However, on multivariate analysis, the effect of this variable was found to be negligible, which suggests that the sample size should probably be increased in order to judge this variable correctly. Furthermore, our data suggest no significant role for NSAIDs in PD, which is in agreement with previous data (Samii et al., 2009).

Night shift workers may have disrupted circadian rhythms and abnormal sleep patterns that lead to a range of physiological, psychological, and social effects. One previous study suggested that working night shifts exerts a protective effect against PD (Chen et al., 2006). In addition to this reported protective effect of shorter sleep duration, these authors also suggested that habitual longer sleep duration may be an earlier marker of PD. Confirming this study, our analysis of nocturnal sleep duration showed that each extra hour of sleep compared to mean sleep duration was associated with a 1.38-fold increase in the chance of developing PD. However, we found, on the basis of our data, that a previous history of evening work increased the risk of PD by 4.6 times $(\mathrm{OR}=4.6$, $95 \% \mathrm{Cl}=1.29-16.86, \mathrm{p}=0.019$ ), a result that, instead, differs from what was found by Chen et al. (2006). Again, better judgments could be achieved by increasing the sample size.

Several studies have evaluated the effect of coffee and tea intake on PD. Caffeine is known as an adenosine receptor antagonist and it is suggested that it may exert a neuroprotective effect (Ross and Petrovitch, 2001; Schwarzschild et al., 2006). The findings of the present study suggest that each cup of coffee per week decreases the risk of PD by $50 \%$ and seemed to show that coffee consumption exerts a protective effect against $P D$, which is consistent with the results of other studies (Ragonese et al., 2003; 2008). Furthermore, in agreement with other studies (Barranco Quintana et al., 2009; Tanaka et al., 2011), this study showed that tea intake exerted a protective effect against PD. Our results suggest that each glass of tea reduced the risk of PD by 0.8 times. It should be taken into account that tea is consumed far more commonly than coffee amongst the Iranian population. Therefore, to obtain a better judgment on the effect of coffee intake on PD onset/progression in the Iranian population we would need to investigate a larger sample.

The effect of adiposity on PD has been investigated in very few studies, which have given conflicting results. For instance, in a case-control study a non-significantly reduced risk of $P D$ was reported with increasing BMI prior to the onset of PD (Ragonese et al., 2008). On the other hand, a large cohort study (Hu et al., 2006) suggested an increased risk of PD with increasing BMI. There are also other studies that show adiposity and high $\mathrm{BMI}$ to be associated with a higher risk of PD (Abbott et al., 2002; Hu et al., 2006). The results of the present study suggest that $P D$ patients have a significantly greater BMI than controls. Unfortunately, numerous overlapping factors can affect BMI, making it difficult to conclude that there is a strong relationship 
between BMI and PD.

Since the 1980s, several studies have evaluated the relationship between agricultural toxins (including pesticides, herbicides, insecticides and fungicides) and PD (Wirdefeldt et al., 2011). It is believed that pesticides can exert neurotoxic effects on dopaminergic neurons. Epidemiologically, there is adequate evidence to suggest that occupational exposure to herbicides and pesticides can increase the risk of PD (Semchuk et al., 1992; 1995; Priyadarshi et al., 2000; Wirdefeldt et al., 2011). A meta-analysis has confirmed this increased risk with a combined OR of 1.94 $(95 \% \mathrm{Cl}=1.49-2.53)$ for all the studies, and $2.15(95 \%$ $\mathrm{Cl}=1.14-4.05)$ for studies performed in the United States (Priyadarshi et al., 2000). Univariate logistic regression analysis of our data suggested that agricultural toxin exposure increases the risk of PD by 1.8 times. This result is in line with the findings of other studies (Semchuk et al., 1992;1995; Kamel et al., 2007), but was not significant according to the chisquare test. Thus, future investigations would require a larger sample size in order to better evaluate this variable.

One of the most widely studied lifestyle habits in relation to PD is smoking. The nicotine in cigarette smoke is believed to stimulate dopaminergic neurons, ameliorate PD symptoms and exert a neuroprotective effect (Wirdefeldt et al., 2011). Several studies have reported an inverse association between smoking and PD (Liou et al., 1997; Werneck and Alvarenga, 1999; Tan et al., 2003; Scott et al., 2005), whereas there are other studies, which do not suggest any notable association (Jiménez-Jiménez et al., 1992; Kuopio et al., 1999; Elbaz et al., 2000). Our data showed no association of smoking with PD in Iranian PD patients. Although there are studies revealing a protective effect of smoking on PD, various systemic biases should be taken into account when evaluating these investigations. For instance, PD patients are more likely to quit smoking. In addition, mortality in smokers could be due to causative factors that are unrelated to PD (Wirdefeldt et al., 2011).

For many years brain trauma has been thought to play a role in initiating or hastening the molecular cascades involved in degenerative disorders including PD (Daneshvar et al., 2011). One study showed that PD patients are more likely to have a history of trauma, possibly because of their progressive motor problems (Rugbjerg et al., 2008). In this study, we found that head trauma did not significantly contribute to the onset or progression of PD.

Epidemiological studies do not provide compelling evidence for the role of hypertension and heart disease in the etiology of PD. In parallel, the results of this study showed no significant differences in hypertension and hyperlipidemia between the PD patients and controls. One study showed a reduction of vascular risk factors in PD patients versus controls (Scigliano et al., 2006).

In conclusion, PD appears to have a complex etiology, involving both genetic and environmental factors. Our results showed that tea and coffee consumption, and short sleep duration probably had a protective role against PD, while no association was found between smoking, toxin exposure, or use of NSAIDs and PD. In this study, evening work and major stressful events markedly increased the risk of PD.

Our study has a number of strengths. It is the first case-control study to assess the relationship between non-genetic and environmental factors and development of PD in Iranian patients. The referral bias in this study is considered to be minimal; the Shariati Hospital has a general neurology clinic, which is neither a tertiary care academic center nor a specialized movement disorders center. Therefore, many of the patients who were referred to this center were patients at Hoehn and Yahr stages 1 or 2 of PD who might be admitted to any neurological center. This clinic is located in the city center and accessible to all members of the population, of all socioeconomic backgrounds.

Our study does present several limitations, however, the first being the possibility of a selection bias: the individuals in the control group, who were in a postoperative state, were chosen only if they were conscious, collaborative and willing to participate. As already mentioned, three controls had to be excluded after refusing to continue with the study. The other possible bias is a participation bias: many PD patients with more advanced disease (based on the Hoehn and Yahr stages) may, because of their movement difficulties, be less likely to attend hospital or a clinic. Finally, because data was collected through patient interview, there is also a possibility of recall bias in this study. Another limitation is the sample size; as already indicated, a larger sample size would probably have allowed a better assessment of the variables. Further epidemiological studies should use larger samples; furthermore, these should include females, so as to better assess hormonal effects in PD.

\section{Acknowledgement}

We would like to thank Dr Esfahani for her valuable assistance in designing the methodology and analysis of the data.

\section{References}

Abbott RD, Ross GW, White LR, et al (2002). Midlife adiposity and the future risk of Parkinson's disease. Neurology 59:1051-1057.

Barranco Quintana JL, Allam MF, Del Castillo AS et al (2009). Parkinson's disease and tea:A quantitative review. J Am Coll Nutr 28(1):1-6.

Benedetti MD, Maraganore DM, Bower JH, et al (2001). Hysterectomy, menopause, and esterogen use preceding Parkinson's disease: an exploratory case-control study. Mov Disord 16:830-837.

Bower JH, Maraganore DM, Peterson BJ, et al (2006). Immunologic diseases, anti-inflammatory drugs, and Parkinson disease: a case-control study. Neurology 67:494-496. 
Chen H, Zhang SM, Hernán MA, et al (2003). Nonsteroidal antiinflamatory drugs and the risk of Parkinson disease. Arch Neurol 60:1059-1064.

Chen H, Schernhammer E, Schwarzschild MA, et al (2006). A prospective study of night shift work, sleep duration and risk of Parkinson disease. Am J Epidemiol 163:726-730.

Daneshvar DH, Riley DO, Nowinski CJ, et al (2011). Long-term consequences: effects on normal development profile after concussion. Phys Med Rehabil Clin N Am 22:683-700.

Elbaz A, Manubens-Bertran JM, Baldereschi M, et al (2000). Parkinson's disease, smoking, and family history. EUROPARKINSON Study Group. J Neurol 247:793-798.

Hancock DB, Martin ER, Stajich JM, et al (2007). Smoking, caffeine and nonsteroidal anti-inflamatory drugs in families with parkinson disease. Arch Neurol 64:576-580.

Hernán MA, Takkouche B, Caamaño-Isorna F, et al (2002). A meta-analysis of coffee drinking, cigarette smoking, and the risk of Parkinson's disease. Ann Neurol 52:276-284.

Holmes TH, Rahe RH (1967). The Social Readjustment Rating Scale. J Psychosom Res 11:213-218.

Hu G, Jousilahti P, Nissinen A, et al (2006). Body mass index and the risk of Parkinson disease. Neurology 67:1955-1959.

Jancovic J, Shannon KM (2008) Movement disorders. In: Bradley WG, Daroff RB, Fenichel GM, et al. (Eds) Neurology in Clinical Practice. Philadelphia, Butterworth-Heinemann, pp. 1762-1802.

Jiménez-Jiménez FJ, Mateo D, Giménez-Roldan S (1992). Premorbid smoking, alcohol consumption, and coffee drinking habits in Parkinson's disease: a case-control study. Mov Disord 7:339-344.

Kamel F, Tanner C, Umbach D et al (2007). Pesticide exposure and self-reported Parkinson's disease in the agricultural health study. Am J Epidemiol 165:364-374.

Kuopio AM, Marttila RJ, Helenius H, et al (1999). Environmental risk factors in Parkinson's disease. Mov Disord 14:928-939.

Liou HH, Tsai MC, Chen CJ, et al (1997). Environmental risk factors and Parkinson's disease: a case-control study in Taiwan. Neurology 48:1583-1588.

Priyadarshi A, Khuder SA, Schaub EA, et al (2000). A metaanalysis of Parkinson's disease and exposure to pesticides. Neurotoxicology 21:435-440.

Ragonese P, Salemi G, Morgante L, et al (2003). A case-control study on cigarette, alcohol. and coffee consumption preceding Parkinson's disease. Neuroepidemiology 22:297-304.

Ragonese P, D'Amelio M, Callari G, et al (2008). Body mass index does not change before Parkinson's disease onset. Eur J Neurol 15:965-968.

Rocca WA, Bower JH, Maraganore DM, et al (2008). Increased risk of parkinsonism in women who underwent oophorectomy before menopause. Neurology 79:200-209.

Ross GW, Petrovitch H (2001). Current evidence for neuropro- tective effects of nicotine and caffeine against Parkinson's disease. Drugs Aging 18:797-806.

Rugbjerg K, Ritz B, Korbo L, et al (2008). Risk of Parkinson's disease after hospital contact for head injury: population based case-control study. BMJ 337;a2494.

Samii A, Etminan M, Wiens MO, et al (2009). NSAID use and the risk of Parkinson's disease: systematic review and metaanalysis of observational studies. Drugs Aging 26(9):769779.

Shwarzschild MA, Agnati L, Fuxe K, et al (2006). Targeting adenosine A2A receptors in Parkinson's disease. Trends Neurosci 29:647-654.

Scigliano G, Musicco M, Soliveri P, et al (2006). Reduced risk factors for vascular disorders in Parkinson disease patients: a case-control study. Stroke 37:1184-1188.

Scott WK, Zhang F, Stajich JM, et al (2005). Family-based case-control study of cigarette smoking and Parkinson disease. Neurology 64:442-447.

Semchuk KM, Love EJ, Lee RG (1992). Parkinson's disease and exposure to agricultural work and pesticide chemicals. Neurology 42:1328-1335.

Semchuk KM, Love EJ (1995). Effects of agricultural work and other proxy-derived case-control data on Parkinson's disease risk estimates. Am J Epidemiol 141:747-754.

Shulman LM (2002). Is there a connection between estrogen and Parkinson's disease? Parkinsonism Relat Disord 8:289-295.

Simon KC, Chen H, Schwarzschild M, et al (2007). Hypertension, hypercholestrolemia, diabetes and risk of Parkinson disease. Neurology 69:1688-1695.

Tan EK, Tan C, Fook-Chong SM, et al (2003). Dose-dependent protective effect of coffee, tea, and smoking in Parkinson's disease: a study in ethnic Chinese. J Neurol Sci 216:163-167.

Tanaka K, Miyake Y, Fukushima W, et al (2011). Intake of Japanese and Chinese teas reduces risk of Parkinson's disease. Parkinsonism Relat Disord 17:446-450.

Thacker EL, O'Reilly EJ, Weisskopf MG, et al (2007) Temporal relationship between cigarette smoking and risk of Parkinson disease. Neurology 68:764-768.

Ton TG, Heckbert SR, Longstreth WT Jr, et al (2006). Nonsteroidal anti-inflammatory drugs and risk of Parkinson's disease. Mov Disord 21:964-969.

Wahner A, Bronstein JM, Bordelon YM, et al (2007). Nonsteroidal anti-inflamatory drugs may protect against Parkinson disease. Neurology 69:1836-1842.

Werneck AL, Alvarenga H (1999). Genetics, drugs and environmental factors in Parkinson's disease. A case-control study. Arq Neuropsiquiatr 57:347-355.

Wirdefeldt K, Adami HO, Cole P, et al (2011). Epidemiology and etiology of Parkinson's disease: a review of the evidence. Eur J Epidemol 26 Suppl 1:S1-58. 